\title{
-Fluid-structure interaction of a two-dimensional membrane in a flow with a pressure gradient with application to convertible car roofs
}

J.J. Knight, A.D. Lucey \& C.T. Shaw

\begin{abstract}
The flow-induced deformation of a membrane in a flow with a pressure gradient is studied. The investigation focuses on the deformation of aerodynamically loaded convertible car roofs. A computational methodology is developed with a line-element structural model that incorporates initial slackness of the flexible roof material. The computed flow-structure interaction yields stable solutions, the flexible roof settling into static equilibrium. The interaction converges to a static deformation within $1 \%$ difference in the displacement variable after three iterations between fluid and structural codes. Reasonably accurate predictions, to within $7 \%$, are possible using only a single iteration between the fluid and the structural codes for the model problem studied herein. However, the deformation results are shown to be highly dependent on the physical parameters that are used in the calculation. Accurate representation of initial geometry, material properties and slackness should be found before the predictive benefits of the fluid-structure computations are sought. The iterative methodology overcomplicates the computation of deformation for the relatively small displacements encountered for the model problem studied herein. Such an approach would be better suited to applications with large amplitude displacements such as those encountered in sail design or deployment of a parachute.
\end{abstract}

(195 words) 


\section{Introduction}

With the increase in computational power, computer aided engineering approaches (including the coupling of multi-disciplinary approaches) are becoming viable for engineering analyses. Most of the development of fluid-structure interaction has used potential flow theory to account for the forces due to fluid motion. This work has been undertaken for flexible surfaces in a pressure field of zero mean-flow streamwise pressure gradient. This paper reports work that has been performed for a flexible surface in an imposed pressure gradient such as that experienced by a convertible roof of a car.

Much of the development of fluid-structure interaction began with the motion of a simplified thin flexible plate. Kornecki (1978) analysed an infinitely long flat plate on which flapping or flutter type motions were possible. Potential flow was used to determine the pressure, which drives/imbalances the system. These analyses were performed in a zero pressure gradient. Likewise, Carpenter \& Garrad (1986) and Crighton (1989) used simple flexible-wall models and small-amplitude deformations to predict the unsteady aero-elastic behaviour of a system using potential flow in a zero pressure gradient. Guruswamy (1990) used the Euler equations to study the aeroelasticity of wings. This work has been extended to incorporate the Navier-Stokes equations (Guruswamy, 1993, 1996). Balint \& Lucey (2005) also solved the NavierStokes equations in the fluid-structure interaction of a cantilevered flexible plate in a channel. The response of a ship rudder behind a propeller has been investigated by Turnock \& Wright (2000). 
For a membrane, the problem usually becomes non-linear, due to the induced tension term, which is a function of displacement. These non-linear effects have been modelled for flexible surfaces in a flow that has zero mean-flow pressure gradient (Newman \& Goland, 1982). A potential-flow solution is used to determine the local pressure field on the deformed surface. This work was extended by Greenhalgh et al., (1984) and Newman $(1984,1987)$ whereby comparisons with experimental data are made. Smith \& Shyy (1995) used the Navier-Stokes equations to model the flow over a twodimensional flexible surface. Substantial differences were realised over the potential flow theory as expected, due to the incorporation of viscous effects including separation from the curved surface.

Flexible surface modelling of membrane structures is similar to that encountered in sail design in that deformations have low wavenumbers, typically that of the fundamental mode. The application also entails the effects of flow separation, which cannot be addressed by a potential flow solution. However, approximate means of simulating the separation over yacht sails using potential flow calculations have been developed (Cyr \& Newman, 1996). Fiddes \& Gaydon (1996) used a vortex lattice method to calculate the flow past yacht sails. The calculation of viscous flow about yacht sails in two dimensions has been performed by Jackson \& Fiddes (1999). A commercial CFD code has also been employed in sail design to study the interaction between combinations of sails (Hedges et al., 1996). Here, the mesh employed was of fixed geometry, so avoiding the complexity of having to calculate the flow over a surface that deforms.

In a statically stable equilibrium, the pressure difference between the upper and lower 
surface balances the structural restorative forces of pre- and induced tension. For a twodimensional membrane, the tension is constant along its length and the effect of

curvature balances the local pressure coefficient (Newman \& Goland, 1982). For a three-dimensional membrane, at any given point, the combination of curvatures in the two directions with their associated tensions must balance the pressure force.

This paper is laid out as follows. A representative formulation of the current problem is first presented. Thereafter, the computational approach is described beginning with the computed flow about the model. A structural solver that is capable of modelling slackness is used in our approach, which is detailed and validated. The coupling of this to the commercial computational fluid mechanics code and source panel method codes is presented including the software interfacing strategy. The results of the computational methodology are presented for various configurations. Finally, some conclusions are offered.

\section{Problem formulation}

Consider the deformation of a two-dimensional flexible surface subjected to a forcing pressure as shown in Figure 1. According to Lucey et al (1997), the governing equation for a this thin flexible surface is

$$
\rho_{m} h \frac{\partial^{2} w}{\partial t^{2}}+D \frac{\partial w}{\partial t}+B \frac{\partial^{4} w}{\partial x^{4}}-\left(T_{0}+T_{I}\right) \frac{\partial^{2} w}{\partial x^{2}}=\Delta P
$$

where $w(x, t)$ is the wall displacement perpendicular to the $x$ direction, $t$ is the elapsed time, $\rho_{m}$ and $h$ are the material density and thickness. $D$ is a dashpot-type damping 
constant, $B$ is the flexural rigidity, $T_{0}$ and $T_{I}$ are the pre-tension and induced tension terms respectively, whilst $\Delta P$ is the fluid pressure difference between the upper and lower surfaces of the flexible wall. Equation 1 assumes one-dimensional motions of the flexible surface, which is a line in two-dimensional space. A full model would include geometric nonlinearity and a second equation relating horizontal motion of a material point with the fluid shear-stress. This is omitted in the current work as shear stresses are at least an order of magnitude smaller than normal stresses in the present problem, wherein a pressure distribution across the membrane is present.

In the present investigation we are interested in deformations with large (non-linear) amplitudes and the effects of pressure gradients including boundary-layer separation. To make the problem tractable, we follow the approach typical of sail design, for example see Cyr \& Newman (1996), and omit the time-dependence in Equation 1. We also omit the flexural rigidity term from Equation 1 so that the objective is then to solve

$$
-\left(T_{0}+T_{I}\right) \frac{\partial^{2} w}{\partial x^{2}}=\Delta P
$$

using a procedure which iterates towards a deformed static equilibrium. The induced tension, $T_{I}$ is a function of displacement, $w$, so that Equation 2 is non-linear. By using a membrane, the motion of the flexible wall can be characterised solely by the consideration of surface points and thus $w(x)$ defines the boundary of the fluid flow relative to the undeformed membrane surface. The present work is aimed at modelling the fluid-structure interaction of convertible car roofs with the surrounding air flow. In simplifying from Equation 1 to Equation 2, the study of flutter instability about the 
deformed state is precluded. Other wave disturbances could be expected to be quickly damped out by the high levels of structural damping present in real padded flexible roofs. There is also the possibility that unsteady large-amplitude solutions about the undeformed state might exist. However, this outcome would be indicated by the failure of our fixed-point algorithm to converge. Thence, the solution is assumed to be steadystate settling to static equilibrium after a certain period of time, so that the structural restorative forces balance the transmural pressure force applied. Other related aspects of the problem beyond the scope of the research are unsteady effects within the wake and those due to changes in forward speed in the case of a car.

\section{Computational Methods}

\subsection{Flow field computation}

Initially, the computation of the flow field about a model with a rigid roof is validated as a first step, the results from which can then be used with confidence in the computational methodology using a flexible roof. The commercial CFD code StarCD (Computational Dynamics, 1997) is used in this work with MARS discretisation (Computational Dynamics, 1997), the SIMPLE algorithm (Patankar \& Spalding, 1972) and the standard k- $\varepsilon$ turbulence model (Jones \& Launder, 1972) for all of the calculations presented herein. A quarter-scale version of the top half of MIRA reference vehicle (Carr, 1992) was used in this work. This was chosen as it is a simplified shape featuring most of the common flow characteristics around the top of a typical car. This facilitates the validation of our methodology by negating certain geometric complexities such as those of the hood that will affect the development of the flow as it approaches the front windscreen. Thus, the flow around the roof of real cars will not be identical to 
that in our model. However, both model and real car will experience a broadly similar characteristic pressure field. The geometry of the model and characteristic dimensions are shown in Figure 2a. The distribution of cells to achieve a mesh independent solution and reasonably accurate capture of separation from the rear corner of the model (Knight et al, 2000) can be seen in Figure 2b. The mesh used is a structured hexahedral grid, created using a FORTRAN 77 program. Biasing is employed towards the model surface to improve the accuracy of the solution and reduce computational expense. It is also used to smooth changes to resolution between the blocks of cells labelled in Figure 2a.

The domain extends $1.25 \mathrm{~m}$ ahead of the leading edge of the model and $3.75 \mathrm{~m}$ aft. The height of the domain is $1.37 \mathrm{~m}$ which corresponds to the height of the University of Warwick wind tunnel permitting comparison of results with a parallel experimental investigation. The turbulence intensity in the middle of the tunnel was measured as to be $1 \%$. This was used with a constant mean flow velocity of $12 \mathrm{~m} / \mathrm{s}$ as the inlet boundary. The inlet flow speed was measured in the experiment using a pitot-static tube located in the free-stream flow upstream of the model. Standard no-slip and no-flux wall boundary conditions using algebraic law-of-the-wall functions are specified at the tunnel walls, ground board and model surface. Symmetry or slip-wall boundaries are applied at the sides of the domain. The standard outlet boundary is used so that pressures and velocities are calculated as part of the solution.

The velocity vectors of the flow field are shown in Figure $3 \mathrm{a}$ and the pressure distribution along the longitudinal centreline obtained using the base mesh is plotted in Figure $3 b$. An experimental pressure distribution, along the flat section of the roof, 
obtained by Knight et al (2001) at the same Reynolds number of 475,000 is also plotted in Figure 3b. The pressure distributions can be seen to be in close agreement. The computation of pressure is therefore judged to be reasonably accurate and therefore suitable for the two-dimensional fluid structure interaction methodology. It is noted that the level of mesh refinement used in this computation violates the law-of-the-wall constraints that are used in the modelling of near-wall turbulence; this could be overcome at far greater computational expense. However, it is sufficiently accurate for the flow-structure investigation of the present study because normal forces are the dominant source of structural deformation.

In two dimensions, reasonably accurate solutions using Navier-Stokes (NS) solvers can be obtained with a relatively small amount of computer time. Carrying out threedimensional calculations increases the expense if the same degree of discretisation is required. Further expense is incurred when the flow solution for a number of different geometries is required, as is the case with an iterative fluid-structure methodology.

Source panel methods (Hess \& Smith, 1967) provide a potential flow (PF) solution that is much simpler to obtain than a solution for the Navier-Stokes equations. Here the use of a two-dimensional boundary-element (or panel) method using first-order source singularities has also been assessed. A comparison of the coefficient of pressure $(C p)$ between the NS solution already described and two PF solutions is shown in Figure 4. The first PF solution only uses panels to describe the model surface and ground board used in the parallel experimental investigation. The resulting pressure field is symmetric about the roof midpoint because separation is not modelled. An improved PF 
calculation is also shown in Figure 4, where a line of panels is used to model a separated shear layer over the rearscreen of the model as drawn in Figure 2a and seen in the NS solution that gave Figure 3a. However, prior knowledge of the separation and reattachment points is required, and must be obtained from the NS solution.

The results from the modified PF solution and the NS solution are broadly in good agreement. The most notable difference is in the region of separation. Taking this into account, the PF solution could be employed as an alternative to the much slower but more accurate NS solution. In addition, the method can be integrated into a coupling methodology quite easily, when compared to NS solver, as it requires only the surface of the vehicle to be discretised, by-passing the need to create a revised field mesh for each step in the fluid-structure iteration.

\subsection{Structural computation}

A code written in FORTRAN 77 evaluates the dynamic structural response of a membrane in two dimensions that represents the roof shown in Figure 2a. The contour of the membrane is discretised into a user-specified number, $N$, of mass points. Time is used as an iteration parameter to step towards the steady state solution for a given pressure field. Figure 5 summarises the calculation of the dependent variables within the structural code.

Initially, the acceleration at each of the panel endpoints along the surface is determined according to the local pressure, which has been obtained by the fluid solver. The CrankNicholson scheme (Ferziger, 1998) is used to calculate the velocity, which is in turn 
used to calculate the displacement in the same manner. These displacements are used to estimate the overall strain, $\varepsilon$, in the membrane according to Equation 3,

$$
\varepsilon=\frac{1}{L} \sum_{i=1}^{N}\left(\left(1+\left(\frac{\partial w}{\partial x}\right)_{i}^{2}\right)^{\frac{1}{2}}-1\right) \Delta x
$$

where $L$ is the distance between hinge points, $N$ is the number of mass points, $(\partial w / \partial x)_{i}$ is the membrane gradient at mass point $i$ and $\Delta x=L / N$ is the length of each panel, having used a uniform discretisation. The induced tension, $T_{I}$, can then be calculated as the product of the strain and its material properties using

$$
T_{I}=\frac{E h \varepsilon}{1-\gamma^{2}}
$$

where $E$ is the Young's modulus and $h$ the thickness of the material. The Poisson ratio, $\gamma$ is fixed at zero as no cross-coupled terms are present in the current two-dimensional work. The solution is time-marched, recalculating the displacements, velocities and accelerations at each specified timestep using the same pressure field. Finite differences are employed, using central differencing to evaluate the curvature, and backward differencing for the gradient of the membrane, as shown in Equations $5 \mathrm{a}$ and $5 \mathrm{~b}$ respectively; the first is second-order accurate and latter is first-order accurate.

$$
\left(\frac{\partial^{2} w}{\partial x^{2}}\right)_{i}=\frac{w_{i-1}-2 w_{i}+w_{i+1}}{(\Delta x)^{2}} \quad\left(\frac{\partial w}{\partial x}\right)_{i}=\frac{w_{i}-w_{i-1}}{\Delta x}
$$


Both calculations are performed explicitly using values obtained from the previous timestep. End conditions are applied in a hinge-type arrangement, so that the displacement of the mirror node about the hinge is equal to the negative of the node nearest the hinge point. This fixes the curvature and displacement at the membrane endpoints to zero.

The code has been validated to obviate errors in implementation, numerical aspects or invalid assumptions in the physical modelling (Knight, 2003). First, the pressure, damping and pre-tension forces are omitted from Equation 1 and a constant pre-tension is used so that the restorative force of the membrane is proportional to its curvature. Releasing the membrane from a deflection with the shape of the fundamental mode, oscillation ensues. The amplitude of this motion remained within $0.1 \%$ after 17 cycles of oscillation while its frequency matched the analytical solution of the reduced system within $0.02 \%$. The former demonstrates that minimal artificial damping has been included in the process of discretisation while the latter confirms the accuracy of the stiffness and inertial forces of the membrane. The code was then tested with the inclusion of a constant external pressure, damping and the non-linear induced tension, which is calculated as part of the computation at each time step according to Equations 3 and 4. Convergence criteria were added to assess the decay of membrane acceleration to zero. The maximum deflections at the midpoint of the membrane were found to be less than $0.2 \%$ different for the two smallest non-dimensionalised convergence ratios of $10^{-3}$ and $10^{-4}$, which corresponded to a $29 \%$ increase in computational expense. The number of mass points was also varied. Using $N=20$ panels gives a $0.16 \%$ reduction in 
deflection of the midpoint as compared to which results for $N=10$. Further details of the validation, convergence criteria and levels of discretisation can been found in Knight (2003) while comprehensive details of the overall approach can be found in Lucey et al. (1997).

The material is known to sag under its own weight. This effect could be reduced by applying pre-tension. However, an extended length or initial slackness defined in Figure 6 is incorporated in the structural solver to model the initial sag that is present in a real application. The initial slackness $\left(\mathrm{s}_{0}\right)$ incurs an extra length of material $\left(\Delta \mathrm{L}_{\mathrm{i}}\right)$ over the distance between the supports (L). This extra length is primarily caused by the attachment of the membrane to the model and can be evaluated using Equation 3 to determine an artificial value of strain and multiplying this by the distance between the supports. The extension due to aerodynamic loading and initial slackness $\left(\Delta \mathrm{L}_{\mathrm{f}}+\Delta \mathrm{L}_{\mathrm{i}}\right)$ can be approximated in the same way. The real extension of the material $\left(\Delta \mathrm{L}_{\mathrm{f}}\right)$ can then be deduced as the difference of the calculated extensions.

\subsection{Coupling methodology}

A higher level of resolution is required by the fluid solver when compared to that required by the structural solver to achieve the same level of accuracy (Guruswamy, 1993). However, coincident nodes, uniformly spaced, are used at the surface of the model roof for both the structural and fluid solvers. This has further served to simplify the development of the coupling strategy, which is summarised in Figure 7. This is deemed acceptable as the structural solver typically reaches a converged solution two 
orders of magnitude faster than that of a NS solver. The grey and black arrows in Figure 7 describe the paths used in the PF and NS approaches, respectively.

To start the process a mesh of surface panels (for PF) or of volume cells (for NS) is created and read by the flow solver program. The pressure calculated by the fluid solver for the flat roof surface of the model is read by an interface program and converted into a set of nodal forces. By assuming static equilibrium conditions, the structural solver calculates a revised surface shape as shown in Figure 7. This surface shape, together with the shape of the rigid model walls and the ground board, is then used as a no-flux boundary surface for the next iteration of the fluid solver. When NS solutions are calculated, no-slip boundary conditions are also applied and a modified field mesh has to be created. The process iterates calculating revised pressures, forces, and displaced shape of the fabric roof. The computation is terminated if the difference between the roof displacements of successive iterations drops below a prescribed value, which was set at $1 \%$. Changes to the geometry less than this were found to have an insignificant effect on the pressure distribution.

\section{Data used in investigation}

A parallel experimental investigation was conducted to assess the validity of the computation. A quasi two-dimensional model was mounted on a ground board as shown in Figure 2a. Both the model and ground board spanned the width of the wind tunnel. An adjustable flap is hinged to the trailing edge of the ground board. The angle of the flap was adjusted so that the flow was parallel to the ground board upstream of its 
leading edge (Knight, 2003).

The material chosen for the flexible roof was a PVC-coated polyester fabric of $0.29 \mathrm{~mm}$ thickness. The Youngs Modulus (E) of the material was found by Choong (2002) under uni-axial loading to be $2.71 \times 10^{7} \mathrm{~N} / \mathrm{m}^{2}$. The computational simulation requires the mechanical properties of this material, which is the product of these two variables and is referred to as the $E h$ value. The material was selected for its similar characteristics yet lower Young's Modulus to that of a typical outer skin of a convertible car roof. The maximum deformation of the material under its own weight was found to be $3.75 \mathrm{~mm}$ at its midpoint. The Cauchy (or Hooke) number, defined in the present study as $C a=\rho U^{2} L /(E h)$, of the model system used herein at a wind speed of $12 \mathrm{~m} / \mathrm{s}$ is $6.85 \mathrm{x}$ $10^{-4}$. The Cauchy number appropriate to the convertible roof of a real car travelling at $12 \mathrm{~m} / \mathrm{s}(43.2 \mathrm{kph})$ is $7.9 \times 10^{-4}$ and $22.0 \times 10^{-4}$ at $20 \mathrm{~m} / \mathrm{s}(72 \mathrm{kph})$. Thus, there is a sufficient level of dynamic similarity between the scale and full-size systems to suggest equivalence in their fluid-structure interaction.

The structural solver described in Section 3.2 requires the pressure difference between the upper and lower surface of the membrane to provide an accurate description of its deformation. The computation only considered external flow over the upper surface of the ground board and model. The location of the reference pressure cell used in the NS solver is the same as the location of a pitot-static tube in the wind tunnel experiment. The cavity in the experimental model was vented beneath the ground board using a hole of $20 \mathrm{~mm}$ diameter. The pressure in the cavity was measured by a digital manometer relative to the static pressure from the pitot-static tube at various wind speeds and found 
to have a pressure coefficient of -0.095 . This resulted in a cavity pressure relative to that of the pitot-static tube of $-10 \mathrm{~Pa}$ using a wind speed of $12 \mathrm{~m} / \mathrm{s}$. In addition, the pressure was measured at various locations within the cavity and found to only vary by $1 \mathrm{~Pa}$. For simplicity, the interface program assumes the pressure is constant in the cavity and accounts for it by subtracting it from the pressure obtained by the fluid solver.

\section{Results}

\subsection{Fixed-point iteration}

Initial conditions have been incorporated within the two-dimensional structural solver by assuming the slackness to take the form of a half-sine wave. This assumed initial shape is not critical to the results because the structural solver permits the shape of the fluid-loaded equilibrium position to be developed solely on the balance between aerodynamic and structural forces. The same pressure field obtained from experiment shown in Figure 3 was applied and not updated. The resulting increase in deformation of the membrane can be viewed from Figure 8 for varying slackness values at the midpoint of the membrane.

The value of slackness can be seen to have a significant effect on the overall displacement. However, the extension in the material at high values of slackness does not lead to as great an overall increase in vertical displacement when compared to the lower slackness values. This is due to the curvature, which together with induced tension counteracts the fluid forces. Therefore, a larger value of curvature requires a smaller value of tension, thus smaller extension in the material, to counteract the same pressure field. The maximum displacement can be deduced from Figure 8 to be forward 
of the midpoint of the membrane. This is due to the lower pressure found near the front of the membrane which requires higher values of curvature to balance the governing equation.

\subsection{Coupled solutions}

The membrane with no slackness is used first in the coupled solution where the pressure field is recalculated in each iteration. For this simplified configuration the algorithm is found to be robust, yielding a converged solution to the coupled computation. Convergence to within $1 \%$ of displacement at each nodal point is reached after just three iterations for both types of fluid solver. The deformation of the membrane roof after each iteration using the NS fluid solver is plotted in Figure 9. The second and third iterations of the coupling scheme gave a $7 \%$ increase in the overall deformation when compared to the deformation obtained after the first iteration. Figure 9 also shows the calculated shape after three iterations of the coupling scheme when the PF solver with reattached shear layer (the dotted line in Figure 9) is used. The predicted deformations arising from the two types of fluid solver are seen to be similar, but the peak of the final deflection predicted by the $\mathrm{PF}$ solver is $5 \%$ greater than, and lies $15 \mathrm{~mm}$ further downstream to, that of the NS solver prediction. The maximum difference is $7.4 \%$ at the location of $213.5 \mathrm{~mm}$ from the front of the membrane. This is expected from a consideration of the pressure distributions shown in Figure 4.

The PF solution is not as accurate as the NS solution as the effects of viscosity including separation are not accounted for and need to be modelled. The estimated shear layer in the current approach leads to a greater suction over the rear of the roof resulting 
in a higher deformation there. However, reasonable agreement in the overall deformation is shown in Figure 9. Therefore, the PF solver could be used as an intermediate fluid calculation as it can obtain a solution two orders of magnitude faster than one from a NS solution. The PF solver is also more easily fully integrated into a single computational code automating the methodology, which can be more readily applied to a parametric analysis.

\subsection{Parametric investigation}

A parametric study has been performed to assess the sensitivity of the initial slackness and material properties on the overall deflected shape with variation in wind speed. The study is not meant to be a comprehensive investigation covering all parameters in the system. The PF solver was used in the coupling strategy with the structural solver that incorporated a slackness $s_{o}=3.75 \mathrm{~mm}$ that was estimated in the experimental work of Knight (2003). The deformations of the membrane are shown in Figure 10 for various wind speeds.

The deformations are seen to exhibit the same characteristic shape, as expected. The maximum deformations of the membrane are shown in Figures 11 and 12 for variations in slackness ratios and material properties respectively. The initial slackness terms remain fixed at $3.75 \mathrm{~mm}$ when varying material properties, and $E h$ is held fixed at $78,600 \mathrm{~N} / \mathrm{m}$ when varying initial slackness ratios.

The variation in material properties and slackness is seen to have a noticeable influence on the displacement of the membrane. The displacement of the membrane at low wind 
speed is mostly offset by the amount of slackness applied. Also, the displacements are seen in Figure 11 to converge with wind speed. This is due to the curvature of the membrane having a greater value so that a reduced induced tension is required to counteract the pressure. This reduction in tension leads to a reduction in displacement at high wind speed.

Conversely, the displacements are seen to diverge at higher wind speeds when varying the material properties. This is because a greater extension, hence displacement, is required to balance the pressure. Any variation in material properties produces a more significant effect than variation in slackness at high wind speeds and vice versa. For example, at a wind speed of $12 \mathrm{~m} / \mathrm{s}$, the variation in displacement is $+15 \%$ for $36 \%$ decrease and $-7 \%$ for a $27 \%$ increase in the $E h$ value of the material. A $-6 \%$ variation is found using no slackness and $+18 \%$ increase is found when using double the slackness. Therefore, the accuracy of material properties is approximately twice as important as level of slackness at a wind speed of $12 \mathrm{~m} / \mathrm{s}$.

Finally, an approximate theoretical treatment of the system equations is outlined that goes some way to explaining the nearly linear relationship between maximum membrane deformation and wind speed seen in Figures 11 and 12. The present static problem is non-dimensionalised using membrane length and upstream dynamic pressure, hence $x^{\prime}=x / L, w^{\prime}=w / L$ and $\Delta P^{\prime}=\Delta P /\left(\rho U^{2}\right)$, so that the non-dimensional form of Equation 2 is 


$$
-\left[\frac{E h}{\rho U^{2} L}\right]\left[\int_{0}^{1}\left\{\left(1+\left(\frac{\partial w^{\prime}}{\partial x^{\prime}}\right)^{2}\right)^{\frac{1}{2}}-1\right\} d x^{\prime}\right] \frac{\partial^{2} w^{\prime}}{\partial x^{\prime 2}}=\Delta P^{\prime}
$$

in the absence of pre-tension, with the Poisson ratio set to zero, and having used the continuous form of Equation 3. The first (non-dimensional) term on the left-hand side is the inverse of the Cauchy (or Hooke) number and serves as a control parameter for the system solution. The integral term can be simplified by first re-normalising using the maximum deflection of the membrane; thus $w^{\prime \prime}=w^{\prime}\left(L / w_{\max }\right)$. Because $\left(w_{\max } / L\right)$ is small, as evidenced by the numerical results of Figures $8-10$, the integrand can be simplified using a truncated binomial expansion and Equation 6 is then closely approximated by

$$
-\left[\frac{E h}{2 \rho U^{2} L}\left(\frac{w_{\max }}{L}\right)^{2}\right]\left[\int_{0}^{1}\left(\frac{\partial w^{\prime \prime}}{\partial x^{\prime}}\right)^{2} d x^{\prime}\right] \frac{\partial^{2} w^{\prime}}{\partial x^{\prime 2}}=\Delta P^{\prime}
$$

The pressure difference on the right-hand side of Equation 7 comprises contributions from the curvature of the entire car roof and the additional curvature due to the deformation of the membrane. For the simpler system depicted in Figure 1, the latter is just $-\partial^{2} w^{\prime} / \partial x^{\prime 2}$ as can be deduced from Carpenter \& Garrad (1986) and Lucey et al. (1997). Thus, by neglecting the overall shape of the roof, any initial slackness, and recognising that the integral in Equation 7, denoted $I\left(w^{\prime \prime}\right)$ below, is constant for a given shape of deformation, the approximate relationship between deformation amplitude and wind speed is 


$$
w_{\max } \approx\left(\frac{2 \rho L^{3}}{E h I\left(w^{\prime \prime}\right)}\right)^{\frac{1}{2}} U
$$

\section{Conclusions}

Two-dimensional NS and PF simulations have been tailored for the present model problem. The latter is not as good a representation of fluid flow but is significantly more computationally efficient. Flow separation from the curved surface of the model has been accurately captured by adding a shear layer in the PF simulation and increasing the resolution of the mesh used in the NS simulation. An accurate prediction of the pressure distribution was obtained for the model problem and used with confidence in the fluidstructure methodology.

A structural solver has been developed to model the response of a membrane in two dimensions incorporating physical aspects of slackness. The structural solver has been validated using a series of tests and successfully coupled with a PF solver and commercial NS flow solver. The coupling methodology was found to be robust for the two-dimensional model geometry requiring only three cycles to reach static convergence for a less than $1 \%$ difference in membrane shape for all of the cases considered. Static deformation of the membrane is found to be dominant over unsteady deformations. The increase in deformation attributed to a coupled solution (relative to that of a single iteration) for the model problem was found to be $7 \%$. Thus, for engineering purposes, the structural deformation of the membrane roof of the model can be reasonably estimated using the pressure field over a rigid roof. 
In addition, the hybrid PF solver was coupled to the structural solver and found to give a solution within $7.4 \%$ of that obtained using the NS solver. This has been automated into a single code and used for a parametric study to assess the sensitivity of slackness and material properties. Variation of slackness had a reduced effect on the final displacement when increasing wind speed, whereas the change in displacement with variation of material properties was highly dependent on wind speed. At a wind speed of $12 \mathrm{~m} / \mathrm{s}$, the change in material properties was found to have twice the influence in final displacement as that using the same percentage change in slackness.

Although the methodology has been shown to provide accurate results, it overcomplicates the computation of deformation for the relatively small displacements encountered in this work. Nevertheless, the methodology has been proven and has wide applicability to other engineering problems. The approach would be better suited to applications of large amplitude displacements such as that encountered in sail design or employment of a parachute.

\section{References}

Balint, T.S., \& Lucey, A.D., 2005. Instability of a cantilevered flexible plate in viscous channel flow. Journal of Fluids and Structures, 20, pp. 893-912

Carpenter, P.W., \& Garrrad, A.D., 1986. The hydrodynamic stability of flows over Kramer-type compliant surfaces. Part 2. Flow-induced surface instabilities. J. Fluid Mech., 170, pp. 199-232.

Carr, G., 1992. Validation of CFD codes for predicting aerodynamic performance, Automotive Engineer, Oct/Nov, pp. 46-49.

Choong, L.-W., 2002. Numerical formulation and modelling of fabric convertible hoods under aerodynamic loading, M.Phil Thesis, University of Warwick. 
Computational Dynamics Ltd., 1997. Star-CD v3.05 User guide and methodology manual. Computational Dynamics Ltd., London.

Crighton, D.G., 1989. The 1988 Rayleigh medal lecture: Fluid-loading - the interaction between sound and vibration. J. Sound Vib., 133, pp. 1-27.

Cyr, S., \& Newman, B.G., 1996. Flow past two-dimensional membrane aerofoils with rear separation, J. Wind Engineering and Industrial Aerodynamics, 63, pp. 1-16.

Ferziger, J.H., 1998. Numerical methods for engineering applications. 2nd edition. Wiley-Interscience, New York. ISBN: 0471116211.

Fiddes, S.P. \& Gaydon, J.H., 1996. A new vortex lattice method for calculating the flow past yacht sails, J. Wind Engineering and Industrial Aerodynamics, 63, pp. 35-59.

Greenhalgh, S., Curtiss, H.C., \& Smith, B., 1984. Aerodynamic properties of 2D inextensible flexible areofoils, AIAA Journal, 22, pp. 865-870.

Guruswamy, G.P., 1990. Unsteady aerodynamic and aeroelastic calculations for wings using Euler equations, AIAA J., 28 (3), pp. 461-469.

Guruswamy, G.P., \& Byun, C., 1993. Fluid structural interactions using Navier-Stokes flow equation coupled with shell finite element structures, AIAA paper, 93-3087.

Guruswamy, G.P., \& Byun, C.,1996. Wing-body aeroelasticity on parallel computers, $J$. Aircraft, 33(2), pp. 421-428.

Hedges, K.L., Richards, P.J., \& Mallinson, G.D., 1996. Computer modelling of downwind sails, J. Wind Engineering and Industrial Aerodynamics, 63, pp. 95-110.

Hess, J.L., \& Smith, A.M.O., 1967. Calculation of potential flow about arbitrary bodies. Prog. Aeronaut. Sci. 8, pp. 1-138.

Jackson, P.S., \& Fiddes, S.P., 1999. Two-dimensional viscous flow past flexible sail sections close to ideal incidence, Aeronaut. J., 99, pp. 217-225.

Jones, W.P., \& Launder, B.E., 1972. The prediction of relaminarization with a twoequation model of turbulence. Int. J. Heat Mass Transfer, 15, pp. 301-314.

Knight, J.J., Shaw, C.T., \& Lucey, A.D., 2000. Fluid-structure modelling of convertible car roofs. Proceedings of the 3rd MIRA International Vehicle Aerodynamics Conference, Rugby, U.K. 18-19th October.

Knight, J.J., Lucey, A.D., \& Shaw, C.T., 2001. On the aerodynamic loading and deformation of convertible car roofs. SAE paper 2001-01-1269. 
Knight, J.J., 2003. Development of a flow-structure interaction methodology applicable to a convertible car roof, $\mathrm{PhD}$ thesis, University of Warwick.

Kornecki, A., 1978. Aeroelastic instabilities of infinitely long plates, I. Solid Mech. Arch. 3, pp. 281-440.

Lucey, A.D., Cafolla, G.J., Carpenter, P.W., \& Yang, M., 1997. The non-linear hydroelastic behaviour of flexible walls. J. Fluids \& Structures, 11, pp. 717-744.

Newman, B.G,. \& Goland, D., 1982. Two-dimensional inflated buildings in a cross wind, J. Fluid Mech., 117, pp. 507-530.

Newman, B.G., 1984. 2D impervious sails, J. Fluid Mech., 144, p. 445.

Newman, B.G., 1987. Aerodynamic theory for membranes and sails, Progress in Aerospace Sciences, 24, p. 1.

Patankar, S.V., Spalding, D.B., 1972. A calculation procedure for heat, mass and momentum transfer in three-dimensional parabolic flows, International Journal of Heat and Mass Transfer, 15, pp.1787-806.

Smith, R., \& Shyy, W., 1995. Computational model of flexible membrane wings in steady laminar flow, AIAA Journal, 33 (10), pp. 1769-1777.

Turnock, S.R., \& Wright, A.M., 2000. Directly coupled fluid structural model of a ship rudder behind a propeller, Marine Structures, 13, pp. 53-72. 


\section{Figure Captions}

Figure 1. Schematic of the underlying principles used in the problem formulation.

Figure 2. (a) Two-dimensional computational domain with base mesh cell allocation (domain not to scale), and (b) The mesh used for the Navier-Stokes solution of the flow field.

Figure 3. (a) Computed (Navier-Stokes) velocity vectors of the flow field for a wind speed $U=12 \mathrm{~m} / \mathrm{s}$, and (b) Comparison of two-dimensional experimental and computed pressure distributions through the variation of pressure coefficient over the roof of the model car.

Figure 4. Computed pressure distribution over the roof of the model car; comparison of the predictions of potential-flow and Navier-Stokes solvers.

Figure 5. Dataflow diagram used in structural code.

Figure 6. Schematic of membrane initial slackness and deflection under loading.

Figure 7. Displacement coupled algorithm using potential-flow and Navier-Stokes solvers.

Figure 8. Computed deflections of membrane roof for different slackness values. $(E h=78,600 \mathrm{~N} / \mathrm{m}, U=12 \mathrm{~m} / \mathrm{s})$

Figure 9. Computed membrane-deformation profile showing convergence of the NSbased solution and a comparison of this with the PF-based solution. $(E h=78,600 \mathrm{~N} / \mathrm{m}, U=12 \mathrm{~m} / \mathrm{s})$

Figure 10. Computed membrane-deformation profile for various wind speeds.

Figure 11. Variation of maximum membrane deformation with wind speed for various values of initial slackness and fixed material properties, $E h=78,600 \mathrm{~N} / \mathrm{m}$. (The straight lines of best fit have been added to aid interpretation of the data.) 
Figure 12. Variation of maximum membrane deformation with wind speed for various material properties and fixed slackness, $3.75 \mathrm{~mm}$. (The straight lines of best fit have been added to aid interpretation of the data.) 